\title{
Pulsed Phase Thermography Imaging of Fatigue-Loaded Composite Adhesively-Bonded Joints
}

\author{
Peter H. Shin, Sean C. Webb, Kara J. Peters* \\ Department of Mechanical and Aerospace Engineering, North Carolina State University, \\ Campus Box 7910, Raleigh, NC 27695
}

\begin{abstract}
We applied pulsed phase thermography to image and size damage in adhesively bonded joints. Specifically, the initiation and propagation of fatigueinduced damage in single lap joints with carbon fiber epoxy adherends was investigated. Lap joint specimens with various levels of manufacturing defects were fabricated and loaded in low-cycle fatigue. A calibration specimen with artificial defects was used to design a threshold algorithm for sizing of the damaged regions. The dominant failure mode in specimens without manufacturing defects was fiber-failure, whereas joints failing prematurely demonstrated adhesive failure. Imaging of the lap joints after regular number of fatigue cycles revealed that manufacturing defects could be detected and the resulting, imminent adhesive failure could be identified prior to joint failure. Additionally, the extent of this damage could be accurately estimated through the sizing algorithm. Due to the brittle nature of fiber-failure, it could not be detected prior to failure of the joint, however this was not critical, as the goal was identify premature failure of the adhesively bonded
\end{abstract}

\footnotetext{
*Corresponding author:

Email address: kjpeters@ncsu.edu ( Kara J. Peters)
} 
joint.

Keywords:

pulsed phase thermography, adhesively-bonded joints, fatigue

\section{Introduction}

Aerospace vehicle components often demonstrate premature aging during service, such as fatigue cracking and corrosion, which must be detect to ensure their safety. In modern composite airframe materials the critical aging phenomena are often subsurface, presenting new challenges for inspection of aircraft [1]. Once critical inspection location is adhesively-bonded joints. Adhesive bonding of multiple composite laminates (adherends) offers substantial advantages over traditional riveting due to their better distribution of stress across the bonded area, low part counts, and high strength to weight ratio of the loading joint region. In addition, adhesive bonding prevents the introduction of free edges at drilled holes, which can later lead to delamination.

However, care must be taken during the fabrication of adhesively bonded joints since environmental conditions and surface preparations can greatly affect the strength and quality of the final bonded joint [2]. Defects appear in two primary regions after manufacturing, within the adhesive layer and at the adherend-adhesive interface [3]. In the adhesive layer, porosity or voids are often caused by air entrapment during the adhesive layup process and cracks can be introduced due to incorrect mixing or thermal shrinkage during cure. Weak bonding or disbonding can also occur at the adherend-adhesive interface due to surface contamination by grease or a loose oxide layer due 
to poor surface preparation. Such defects often lead to premature failure of the joint before failure of the composite adherends is achieved.

Previous authors have demonstrated that fabrication defects in bonded joints can be easily detected using infrared thermography $[4,5,6]$. The detection and sizing of damage in adhesively bonded joints is particularly well captured by thermographic imaging, as the damage plane is typically perpendicular to the thermal wave propagation direction $[7,8]$. For example, Meola et al [6] demonstrated that surface roughness, artificial defects and grooves could be imaged in adhesively-bonded metallic joints using lock-in thermography. Waugh et al $[4,5]$ applied PPT for imaging of fabrication defects in adhesively-bonded joints, including surface contamination with silicon grease and PTFE inserts. They demonstrated that the PTFE inserts were relatively easy to detect, whereas the silicon grease was only detectable when the joint was under an applied static load, presumably due to opening of the joint during some induced bending. These results highlight the importance of a relatively high thermal contrast between the defect and the surrounding material for successful imaging.

The goal of this paper is to identify whether or not realistic damage modes due to fatigue have sufficient thermal contrast to be observed using infrared thermography. We focus on a small scale representation of an aerospace adhesively-bonded lap joint with fatigue damage due to low frequency cyclic loading. In this work, we will apply pulsed phase thermography (PPT) because it can image large sections of an airframe relatively quickly and is therefore a promising technique for commercial aircraft. Other thermography methods, such as lock-in thermography or frequency modulated 
thermography, could similarly be applied with increased spatial resolution, at the cost of increased imaging times.

Prior to imaging of adhesively bonded joints with fatigue induced damage, we calibrate the PPT phase images using a specimen with polymer inserts. As the specimens were manually fabricated, PPT imaging was applied to both specimens that achieved the full fatigue potential of the joints and specimens that showed premature failure due to manufacturing defects.

\section{Experimental Methods}

This section describes the preparation of adhesively bonded lap joint specimens, the low-cycle fatigue loading protocol applied to them and the PPT setup and parameters for imaging of fatigue induced damage.

\subsection{Specimen Preparation}

All carbon fiber reinforced epoxy adherents were fabricated from 8 layers of pre-impregnated 2 × 2 twill woven carbon fiber (Advanced Composites LTM22/CF0300). Initial laminates were fabricated as $254 \mathrm{~mm}$ x $279.4 \mathrm{~mm}$ sheets with plies aligned in the $0^{\circ}-90^{\circ}$ directions. Prior to the laminate layup, a layer of mylar vacuum bag, two layers of breather ply and a single layer of peel ply were placed on an aluminum plate to prevent excessive resin on the laminate surface. The final assembly was covered by peel ply, breather ply, and finally mylar as the bottom layer. The edge of the vacuum bag was sealed and the air between the two mylar layers drawn out using a vacuum line. An additional aluminum plate was placed on the top and bottom of the mylar sheet to evenly distribute the heat and pressure throughout the curing process. the sample was placed in a hotpress, preheated to $50{ }^{\circ} \mathrm{C}$, and 
pressurized to a constant pressure of 8.27 MPa. The hotpress temperature was then increased every 15 minutes by $15{ }^{\circ} \mathrm{C}$ until it reached $80{ }^{\circ} \mathrm{C}$. The laminate was then cured for an additional 3 hours at $80{ }^{\circ} \mathrm{C}$ and then allowed to cool for 12 hours at room temperature. Once cured, the laminate was cut into individual pieces using a wet tile saw.

Two different specimen geometries were fabricated: a specimen for calibration of the PPT imaging and lap joint specimens for fatigue loading. The calibration specimen was fabricated from two $252 \mathrm{~mm}$ x $101.6 \mathrm{~mm}$ panels, as shown in figure 1. For this specimen, four artificial defects were introduced, created from various layers of $25.4 \mathrm{~mm}$ x $25.4 \mathrm{~mm}$ polytetrafluoroethylene (PTFE) tape and a single layer of polypropylene (PP) tape of the same dimensions. Each layer of PTFE tape had a thickness of $0.089 \mathrm{~mm}$. All defects were spaced $38.1 \mathrm{~mm}$ from one another. Figure 1 shows the bottom laminate with the inserts prior to the application of the adhesive layer.

The second specimen geometry followed the the ASTM D3165 standard for single lap joint specimens for fatigue testing. The dimensions of these specimens are shown in figure 2. This particular single lap joint standard prevents bending of the overlap region during uniaxial loading of the specimen. Bending of the overlap region creates peeling stresses, changing the stress condition from that of an actual structure. In addition, bending would open areas the joint with poor bonding and therefore artificially increase the contrast of thermal images $[4,6]$.

For each specimen, the joining surfaces were prepared by sanding and cleaning each adherend with $\mathrm{Al}_{2} \mathrm{O}_{3} 60$ grit sand paper and isopropyl alcohol. Hysol EA 9394 epoxy paste adhesive was applied to the adherends and dis- 
tributed manually, using a steel blade parallel to the surface. The specimens were then placed between two mylar sheets and placed in the hotpress and cured for one hour at $66^{\circ} \mathrm{C}$. The specimens were cooled at room temperature for 24 hours.

\subsection{Fatigue Loading}

Using an Instron servohydraulic fatigue testing machine, load-controlled, fully-reversed cyclic loading was applied to the composite lap joints with a frequency of $3 \mathrm{~Hz}$ and in blocks of 200 cycles. The peak-to-peak amplitude of the cyclic loading was determined based on an initial tension to failure test of three lap joint specimens, at a displacement controlled loading rate of 0.5 $\mathrm{mm} / \mathrm{min}$. This peak-to-peak amplitude was set at $0.445 \mathrm{kN}$, approximately $13 \%$ of the mean failure load for these three specimens. After each 200 cycle loading block, the lap joint was removed from the fatigue testing machine for pulsed-phase thermography imaging.

A small amount of pre-tensioning was applied to the joint to simulate an in-flight load and the PPT images obtained again. The specimen was then returned to the uniaxial testing machine to continue the fatigue loading. This process was repeated until failure of the lap joint. If the lap joint did not fail after a total of 1000 cycles, the fatigue cycles per loading block was increased to 1000 and the testing continued until specimen failure. After failure of the lap joint, the fracture surfaces were photographed.

\subsection{Pulsed Phase Thermography}

Pulsed phase thermography (PPT) applies a square pulse to heat the sample, from which the thermal response at the front surface (in reflection) is 
decomposed into a multitude of individual thermal waves at different frequencies through Fourier transforms $[10,11]$. In this method only a single pulse heating of the structure is required which significantly reduces the imaging time. Post-processing of phase images from the real and imaginary portions of the Fourier transform at different frequency ranges yields information on subsurface defects and their depth. Details of the analysis procedures can be found in [11].

The experimental setup for PPT imaging is shown in figure 3. Thermal images were acquired using a mid-wave infrared thermal camera, Cedip $560 \mathrm{M}$, with a spectral range of 3.6 to $4.9 \mu \mathrm{m}$ and Indium Anitmonide (InSb) detector. The size of the recorded images were 640 x 512 pixels. The IR camera was installed on an aluminum rail, perpendicular to the plane of the sample, at a distance of $1.16 \mathrm{~m}$ from the specimen surface. The sample was supported by two tension cables which were connected to vertical rails on each side of the sample. A function generator provided a $10 \mathrm{~V}$ square pulse signal for 7 seconds to the IR power module. The IR power module amplified the input signal to power the halogen lamps. Two halogen lamps were used as the heat source, each supplying $1700 \mathrm{~W}$ output power at the $10 \mathrm{~V}$ input signal. The heat sources were positioned at a $30^{\circ}$ angle and $0.5 \mathrm{~m}$ from the specimen surface.

During heating, thermal images of the surface were capture from the camera until 33.5 seconds after the end of heat pulse at a $60 \mathrm{~Hz}$ frame rate. The heating period data and extraneous metadata was removed from the recorded thermal image data files and the remaining image frames saved in MATLAB file format. The image frames were then down-selected to the required sam- 
pling frequency. An open source MATLAB program, IRVIEW, was then used to calculate the phase images from the thermal data [9]. Details on the phase image post-processing will be described in section 3.1.

\section{Results}

\subsection{Calibration}

The calibration sample was used to verify that high contrast damage in the adhesively-bonded joint could be imaged with PPT and to derive a sizing algorithm for the damage based on the measured phase contours. The thickness variations in the adhesive layer and voids due to the manual fabrication method also provided a realistic level of maximum expected noise to the measurements.

The bonded joint thickness was measured after complete cure using a micrometer. The measurements were taken $15 \mathrm{~mm}$ from the lengthwise edges, as seen in figure 1. The measurements are plotted in figure 4 along with a linear fit to the top and bottom thickness. A thickness increase is observed along the length and width of the sample. As the thickness of the adherends did not significantly vary, this thickness increase is due to the adhesive layer. A void was also visible at the edge of the sample near the 4 layer PTFE location. This void is likely to corrupt the phase angle data around this region due to the difference in thermal properties of the air and the composite. These fabrication inconsistencies, such as thickness variations and voids, are typical of those in poorly manufactured joints which can lead to premature failure of the joint. Similar variations were seen in some of the lap joint specimens, although of lesser magnitude due to the smaller overlap area. 
PPT imaging of the calibration sample was then performed. The thermal image measured $26 \mathrm{~s}$ after the end of the heat pulse is shown in figure 5 . As expected, the defects are not highly visible in the thermal image. The diagonal rib of the twill weave appears in the temperature image due to the twill formation between tows and picks in the material. The rate of change in temperature for the defective areas was lower than that of the non-defective area due to their lower thermal effusivity.

Phase images were then calculated from the thermal data using IRVIEW and the phase contrast was defined as the difference between the phase value of each pixel and the overall minimum phase value of the phase image. The phase contrast for the calibration specimen is shown in figure 6 . This phase data was acquired at a sampling frequency of $6 \mathrm{~Hz}$ with a truncation window of $10 \mathrm{~s}$, starting $1 \mathrm{~s}$ after the end of the heat pulse to eliminate the effect of heat saturation [13]. The high phase delay region in the upper right corner overlaps the region with the visible edge void. The approximate locations of the 4 defects are shown with black lines in figure 6 . The maximum phase contrast in the single layer PP tape region was 0.926 rads while that of the single layer PTFE tape was 0.910 rads, due to the difference in thermal conductivity between the two materials (and therefore the difference in thermal contrast).

Phase contrast values, $\Delta \phi$, along the line crossing all four defects are shown in figure 7. A common method to define the boundary between defective and non-defective regions is to calculate the full-width-at-half-maximum (FWHM) [4]. The FWHM is an arbitrary cutoff, therefore it can underestimate or overestimate the size of the data [4]. We will apply the threshold 
concept but use the actual data fork to set the percentage threshold. The data of figure 7 highlights that this FWHM calculation must be localized to the maximum for each individual defect and not the entire region of interest. In addition, the definition of the defect area is difficult since there are no sharp boundaries between the defective and non-defective regions for each defect. The material weave geometry also superimposes a periodic noise on the phase data. Our goals were both to calculate an appropriate threshold value and to determine if a percentage threshold value is even applicable for the different defects with different levels of thermal contrast.

We first calculated the actual boundary region for the two center defects in figure 6 by using the specimen width in figure 5 to calculate the dimensions of each pixel (2.92 pixels $/ \mathrm{mm}$ ). The location of each defect was then mapped onto figure 6 . The maximum pixel phase value was calculated within each boundary and the average phase value of the pixels in the boundary were calculated for each defect. The data for each defect is shown in table 1.

Of the four defects shown in figure 6 , the two center defects were considered more reliable data. The 2 layer PTFE tape region (shown on the right) was influenced by the void at the edges of the sample. The PP tape (shown on the left) was at the extreme left hand boundary of the image and was therefore influenced by boundary effects in the phase calculation. We therefore defined the boundary threshold, based on the average of the two center defects, to be $72.5 \%$. It is encouraging that the values were actually quite close between the four defects, even with the fabrication variations across the specimen. We then applied the threshold criterion and mapped pixels in the phase image of figure 6 that met this criterion. These results are shown in 
figure 8. In order to highlight the ability of a single threshold value to size both detects, the threshold criterion was applied only in the regions immediately surrounding the two defects center. Pixels outside of these regions of interest where mapped to white.

The threshold binary image well defines the damage boundaries for the two defects. The surface weave pattern is clearly visible in figure 8 , however this effect could be mitigated through image filtering, as the weave pattern is periodic. While the results of figure 8 are promising, the context of these results must be understood. The defects in this specimen are artificial with sharp edges and therefore it is not clear how the threshold algorithm will transition to fatigue induced defects. The use of a local maximum also presents challenges because it is based on phase contrast across the specimen, rather than an absolute value. Initially when there is not much damage present, the contrast would be low, causing a large portion of the region of interest to be identified as damage. A user-defined threshold to consider that an actual damage has occurred would be required. We will consider both of these issues in the next section.

\subsection{Fatigue Damage}

As discussed in section 2.2, final nine single lap joint specimens were subjected to fatigue loading. The fatigue loading was interrupted at regular intervals, and PPT images obtained of the joint region. The analysis of these images was driven by the results from the calibration specimen discussed in the previous section.

To determine the optimal processing parameters for the PPT measurements, thermal images were collected from the same single-lap joint specimen 
(prior to fatigue loading) at three different locations. The same thermal pulse magnitude and duration were used as for the calibration specimen. All three regions reached thermal equilibrium after 33.5 secs. The PPT acquisition parameters were then fixed to begin $1 \mathrm{sec}$ after the end of the thermal pulse for a duration of $32.5 \mathrm{sec}$. Including a time delay before the acquisition prevents errors due to temperature saturation of the IR camera image immediately after heating [14].

Using these acquisition parameters yielded a frequency resolution of $\Delta f=$ $0.031 \mathrm{~Hz}$ for the lap joint specimens. Changing the PPT acquisition parameters was expected to change the calibration process, therefore the calibration tests presented in section 3.1 were repeated using the updated PPT acquisition parameters. The average percentage ratio of the damage boundary pixel phase contrast value to the maximum phase value for the artificial defects was $66.2 \%$ for these acquisition parameters. Thefore the image threshold of $66.2 \%$ of the maximum phase contrast value will be applied to the lap joint specimens.

Figure 9 shows phase images of three of these samples, immediately after fabrication of the lap joints and prior to any loading. The uniformity of the phase images highlights the wide difference in fabrication quality due to the manual fabrication. To discuss the efficacy of PPT imaging for these tests, we will present results from these three particular representative specimens, based on their different levels of fabrication quality and resulting failure mechanisms. Notations for each specimen were made on the surface with a reflective marker. Depending upon the direction from which the specimen was imaged, these marker lines appeared as regions of low or high phase in 
the PPT phase images.

Specimen 3 was one of the highest quality specimens fabricated, showing no regions of high phase contrast in the overlap joint area (in figure $9(\mathrm{c})$ ). The bonded region of the joint is marked by the red box in each image. The only regions of potential poor quality were located on the left side of the specimen, entirely with the loading grip region. This phase contrast is probably due to poor resin flow and/or insufficient pressure during cure of the specimen. However, this potential defect was not expected to reduce the fatigue life of the specimen since it was entirely located in the grip region during the fatigue loading. This was supported by the fact that specimen 3 survived over 50,000 fatigue cycles prior to failure.

The phase image of specimen 1 is shown in figure 9 (a). This specimen was considered of medium fabrication quality, based on the presence of some regions of high phase contrast (in the left hand side of the image). While the majority of the poor quality region was located in the specimen section in the grips during fatigue loading, an initial defective area is seen in the lower portion of the bonded joint. Specimen 1 survived for 1600 cycles of fatigue loading, failing during pre-tensioning after these cycles.

Specimen 2 was considered a specimen of low quality, as can be seen by the large amount of initially high phase contrast regions in figure 9 (b). This initial observation from the PPT images was confirmed by the fact that specimen 2 failed prematurely after 600 fatigue cycles.

After fatigue loading to failure, the two interior surfaces of each lap joint were photographed. These photographs are shown for specimens 1, 2, and 3 in figure 10. Specimen 3 failed through a combination of fiber-tear failure and 
light-fiber-tear failure (as shown in figure 10 (c)). In other words, the adhesively bonded joint reached its full loading capacity since the failure was due to fatigue wear of the carbon fiber epoxy adherends. In contrast, specimen 1 failed through a combination of adhesive failure and light-fiber-tear failure. Regions where the adhesive failed can be seen in figure 10 (a). The amount of premature adhesive failure was even greater for specimen 2 which also failed through a combination of adhesive failure and light-fiber-tear failure (see figure $10(\mathrm{~b})$ ). These results are consistent with the fabrication quality indications from the initial PPT images for each specimen. The presence of different failure modes in the three specimens permits us to investigate the ability of PPT imaging to detect different fatigue-induced, imminent failure modes in adhesively bonded joints, in particular premature adhesive failure.

We now discuss the PPT imaging results for each specimen. The first step in the process was to define the minimum phase value in the region of interest to calculate the phase contrast. The region of interest was first determined by scaling the width of the specimen to the known width for each image (as the specimen was removed from the test fixture between each image) and calculating the pixels in the adhesive bond region. Part of the difficulty in automating the entire process is the pixels with low phase values due to extraneous features such as the writing on the surface or the sudden change in thickness of the specimen and the beginning of the overlap region (as seen in figure 9 (b)). Similar issues would be expected in field applications of the technique due to contaminants on the surface of the structure or surface defects. We therefore allowed the user to select multiple points within the adhesive joint region with the minimum phase values observed. An average 
the $5 \times 5$ pixel area each point was calculated and then averaged between the three points to set the minimum phase value. For these experiments, three points were selected. Figure 11 shows the phase contrast calculated for the adhesive joint region of specimen 1 after fabrication using two separate user choice of minimum phase value points. The negligible difference between the two images shows that the user input did not significantly affect the PPT phase contrast calculations.

We first consider specimens 1 and 2, which prematurely failed due to a combination of adhesive failure and light-fiber-tear failure. The PPT phase images acquired after different numbers of fatigue cycles are shown figures 12 and 13 for specimens 1 and 2 respectively. For each specimen, one PPT phase image acquired during tensioning of the specimen in between fatigue loading cycles are also shown. As expected, the phase contrast in the PPT images generally increased with increasing number of fatigue cycles and the damage initially present in the adhesive bond grew as a function of fatigue loading for specimen 1. However it is interesting to note that the PPT image obtained during tensioning of the specimen (see figure 12 (c)) showed an increased phase contrast as compared to that after the same number of fatigue cycles, but without the applied tension (figure 12 (b)). It was verified that this phase contrast increase was removed once the tension was released. The cause of this damage size is not known, however it is speculated that tensioning the specimen may further propagate cracks in the adhesive layer. Tensioning of the specimen may also have opened existing cracks slightly, increasing the thermal contrast of the damage and therefore the phase contrast in the PPT image. 
Similar results can be seen in the PPT images obtained from specimen 2, although there was not a large increase in phase contrast over the specimen lifetime as a significant defect was present in the adhesive prior to fatigue loading. The size of this defect grew slighlty as seen in figure 13 (d). Interestingly, applying tension to the specimen after 400 fatigue cycles (see figure 13 (c)) did not increase the phase values in the PPT image, but did increase the local phase contrast near the defect as compared to the untensioned case of figure 13 (b).

In contrast, the PPT images acquired from specimen 3, which did not prematurely fail, did not show significant changes over the lifetime of the specimen. PPT images obtained after up to 50,000 fatigue cycles are shown in figure 14. No significant differences appear between the PPT images. These images are consistent with the photograph in figure 10(c), confirming that the failure mode was a rapid, brittle failure with no significant adhesive failure prior to the final fracture of the bonded joint.

\subsection{Threshold Sizing}

Finally, once the maximum phase value within the adhesive bond region was determined for each image, a threshold factor of $66.2 \%$ was applied to the region of interest, following the procedure of the calibration specimen. Binary images were generated of the damaged and undamaged regions. Binary sizing images after selected cycles are shown in figures 15 - 17 for specimens 1, 2 and 3 respectively.

For specimen 1 the preexisting defects appear as a small defect in the threshold image of 15(a). As the number of fatigue cycles increased, this initial defect disappeared from the threshold images for specimen 1. A clear 
indication of fatigue damage did not appear until 1000 cycles (15(e)) and was present until failure of the specimen. The fact that the defects disappear is not an artifact of the binary imaging performed to generate the images in figures 15 and 16, but is actually present in the original measurements. The same areas detected as defects prior to loading of the specimens 1 and 2 can be seen in the phase contrast images of figures 9(a) and (b) (but not in 9(c) for specimen 3). These regions of differing phase contrast then does not appear in the image after 200 cycles of loading, figure 12(a) for specimen 1. After an increased number of fatigue cycles $(>600)$, the same region begins to be highlighted again in figures 12 (c-e). It is therefore thought that some defect is present in this region resulting from the fabrication process, for example due to an air pocket. The thermal contrast at this defect was reduced by the initial loading of the specimen, perhaps due to a release of residual stresses in the bond at this location. However, after continuous fatigue cycles this defective area appeared again and was eventually the source of failure for the bonded joint.

A similar behavior can be seen in the threshold images from specimen 2 in figure 16. In this case, the initial defect appeared to grow and decrease slightly until late in the specimen lifetime. Interestingly, the addition of a preload increased the apparent size of the defect in specimen 2, but not in specimen 1 (figures 16(d) and 15(d)). Pre-tensioning does not appear to be the cause of the growth of the detected defect, as this did not correlate between specimen 1 and 2. In contrast no localized defective regions in the adhesive joint overlap region appeared in the threshold images of specimen 1 prior to failure. All detected pixels where in the noise level of the measure- 
ments since the maximum phase contrast value was close to the minimum phase contrast value.

These threshold images highlight the fact that PPT can identify the presence of manufacturing defects, which lead to premature adhesive failure, once they reach a critical size with respect to fatigue loading. The presence of the manufacturing defects was clear for a wide range specimens in the PPT phase images, but was less consistently identified in the threshold images. The binary sizing images and PPT images indicate that the light-fiber-tear and fiber-tear failure modes cannot be detected with PPT. Both of these failure modes are brittle failure modes, occurring rapidly once initiated. It is therefore reasonable to assume that these brittle failure modes did not begin until after 50,000 fatigue cycles for this specimen. However, it is these failure modes which are considered in design analyses for such joints and are therefore accounted for in fatigue life predictions. Therefore, PPT images can identify adhesively bonded joints which will fail prematurely due to existing defects or adhesive failures.

\section{Conclusions}

This study investigated the ability of PPT imaging to detect critical failure modes in adhesively bonded joints, subjected to fatigue loading, and the use of a threshold algorithm to size the defects. The critical failure modes observed in the lap joint specimens subjected to fatigue loading were adhesive failure, debonding phenomenon between adherend and adhesive, and lightfiber-tear failure due to weak surface bonding. For the calibration specimen,

a threshold value based on the ratio between the maximum phase value of 
defects and average phase value of the boundary of inserted defects, provided a consistent threshold value between various defects. The variation between defects with various thicknesses and noise levels was negligible. Applying this technique to the lap joint specimens before and after fatigue loading demonstrated that the threshold technique highlighted the presence of de-

fects that would lead to premature failures. In contrast, PPT imaging of the sample with a relatively high quality of sample manufacturing showed no indication of damage in the threshold plots. The fracture surface of this sample presented strong characteristics of fiber tear failure, leading to brittle failure and a fully achieved fatigue life of the joint.

\section{Acknowledgements}

The authors would like acknowledge the financial support of the National Science Foundation (CMMI 0900369) for this work.

\section{References}

[1] Boller C, Staszewski WJ. Aircraft structural health and usage monitoring. In: Staszewski WJ, Boller C, Tomlinson GR, editors. Health monitoring of aerospace structures: Smart sensor technologies and signal processing, Chichester, England: Wiley Inc.; 2004, p. 29-74.

[2] Ashcroft IA, Hughes DJ, Shaw SJ. Adhesive bonding of fibre reinforced polymer composite materials. Assembly Automation 2000;20(2)150-61.

[3] Quaresimin M, Ricotta, M. Fatigue behavior and damage evolution of single lap bonded joints in composite material. Composites Science and Technology 2006;66(2)176-87. 
[4] Waugh RC, Dulieu-Barton JM, Quinn S. Thermographic identification of defects in adhesively bonded joints. Proceedings of SEM Annual Meeting 2013.

[5] Waugh R, Dulieu-Barton JM, Quinn S. Pulse phase thermography and its application to kissing defects in adhesively bonded joints. Applied Mechanics and Materials 2011;70:369-74.

[6] Meola C, Bruzzone A, Giorleo L, Morace RE, Vitiello A. Application of lock-in thermography in nondestructive evaluation of adhesivelybonded aluminum joints. Journal of Adhesion Science and Technology 2004;18(6)635-54.

[7] Hobbs C, Temple A. The inspection of aerospace structures using transient thermography. British Journal of Non-Destructive Testing 1993;35(4)183-9.

[8] Türler D. Predicting the geometry and location of defects in adhesive and spot-welded lap joints using steady-state thermographic techniques. Proceedings of SPIE, Thermosense XXI 1999;3700:54-62.

[9] Klein MT, Ibarra-Castanedo C, Maldague XP, Bendada A. A straightforward graphical user interface for basic and advanced signal processing of thermographic infrared sequences. Proceedings of SPIE, Thermosense XXX 2008;6939:14-1-14-9.

[10] Maldague XP, Marinetti S. Pulse phase infrared thermography. Journal of Applied Physics 1996;79(5)2695-8. 
[11] Maldague XP, Galmiche F, Ziadi A. Advances in pulsed phase thermography. Infrared Physics and Technology 2002;45(3-5)175-81.

[12] Webb S, Shin P, Peters K, Zikry MA, Stan N, Chadderdon S, Selfridge R, Schultz S. Characterization of fatigue damage in adhesively bonded lap joints through dynamic, full-spectral interrogation of fiber Bragg grating sensors - part 1, experiments. Smart Materials and Structures 2014;23(2):025016.

[13] Ibarra-Castanedo C. Quantitative subsurface defect evaluation by pulsed phase thermography: depth retrieval with the phase. University of Laval, Dissertation 2005.

[14] Ibarra-Castanedo C. Pulsed phase thermography inversion procedure using normalized parameters to account for defect size variations. Proceedings of SPIE, Thermosense XXVII 2005;5782:334-341. 
Table 1: Phase contrast $(\Delta \phi)$ statistics for each defect in calibration sample.

$\begin{array}{cccc}\text { PP } & \text { PTFE } & \text { PTFE } & \text { PTFE } \\ & \text { (1 layer }) & \text { (2 layers }) & \text { (4 layers })\end{array}$

\begin{tabular}{lcccc} 
maximum $\Delta \phi$ (rads) & 0.116 & 0.099 & 0.156 & 0.139 \\
boundary average $\Delta \phi$ (rads) & 0.091 & 0.074 & 0.123 & 0.098 \\
average threshold $(\%)$ & 78.5 & 74.7 & 78.9 & 70.5 \\
\hline
\end{tabular}




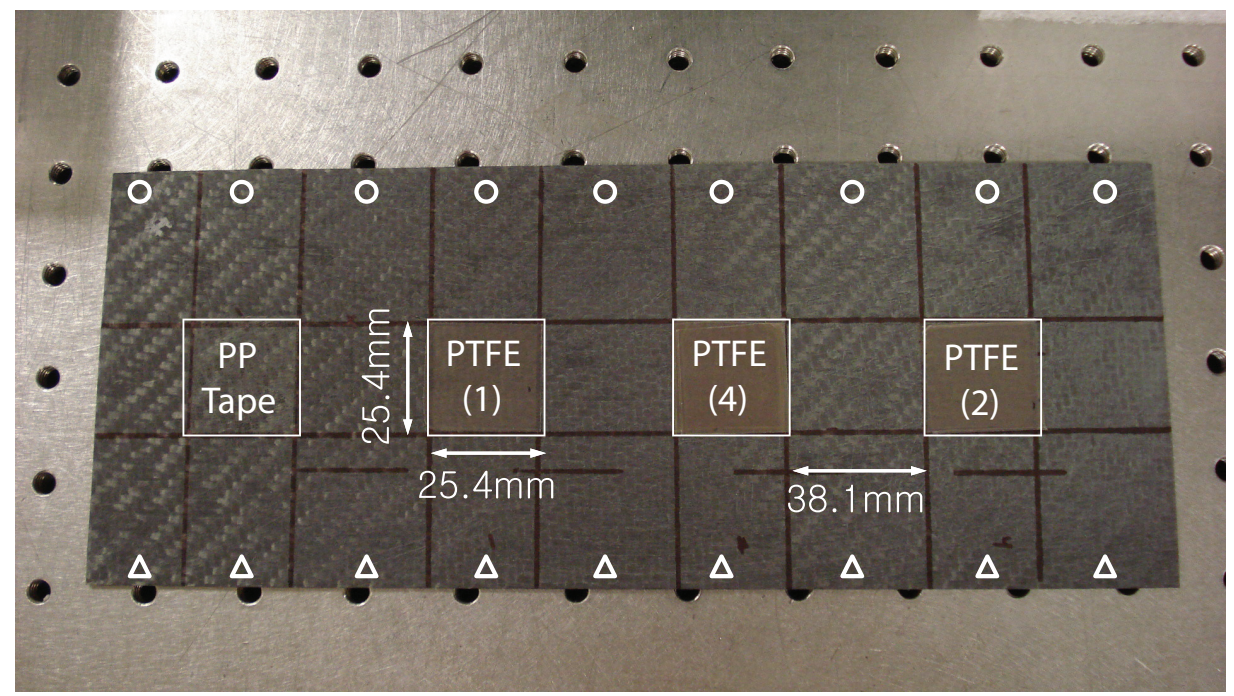

Figure 1: Location of simulated defects on joining surface of bonded joint. Number of PTFE tape layers is indicated in parentheses. Location of thickness measurements plotted in figure 4 are also shown. 

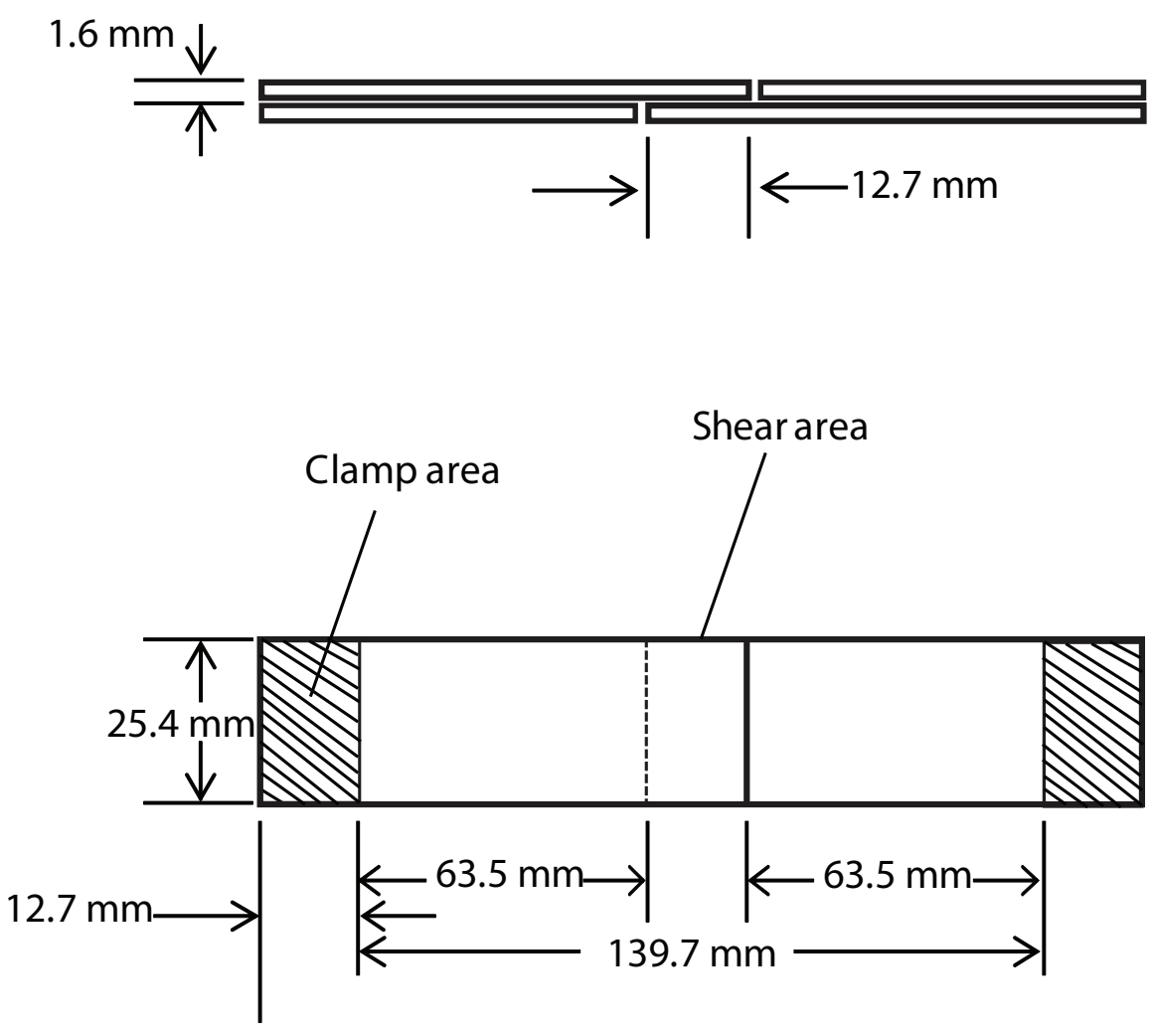

Figure 2: Dimensions of composite lap joint specimens based on ASTM D3165. 


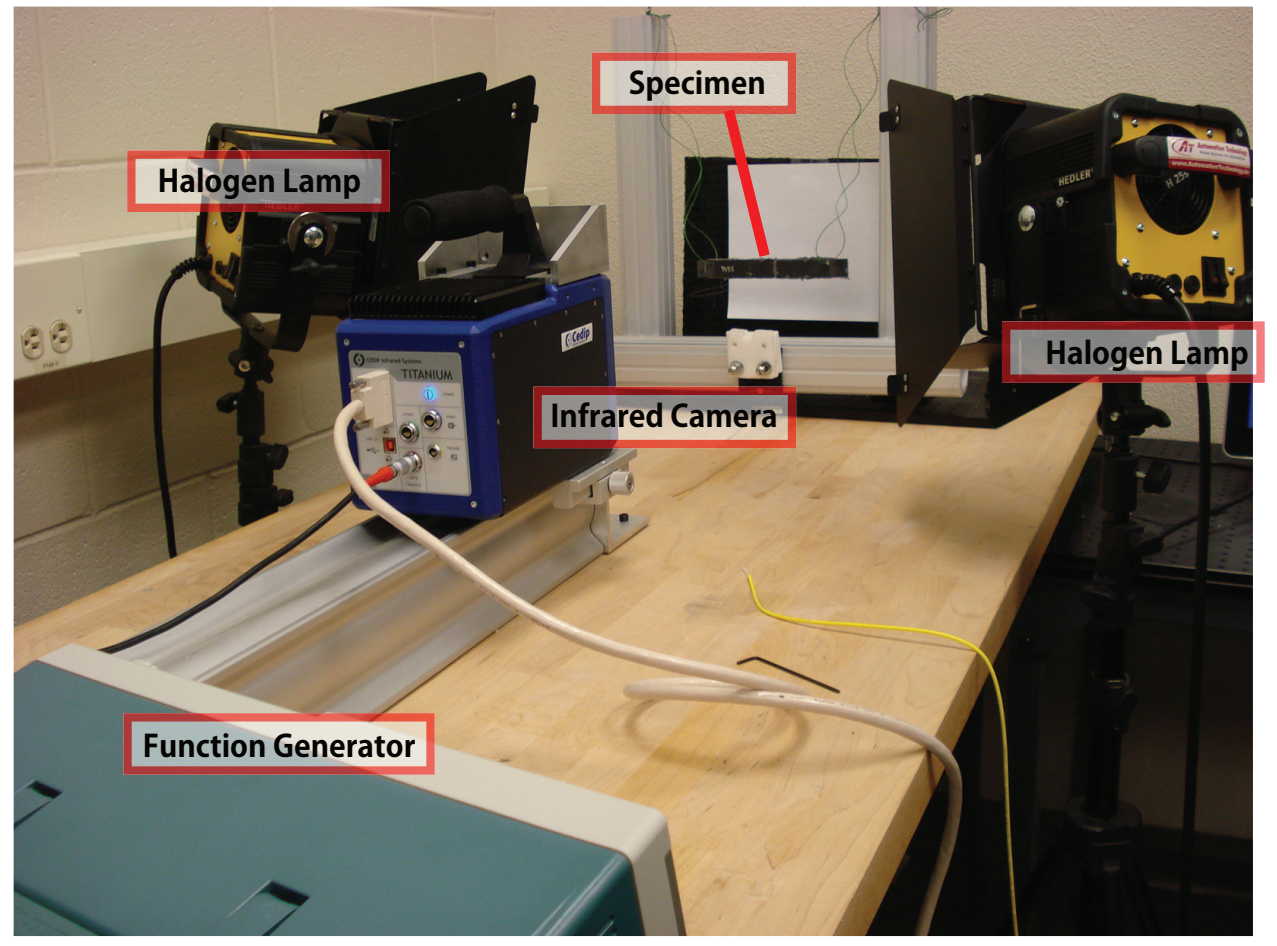

Figure 3: Pulsed phase thermography experimental setup. 


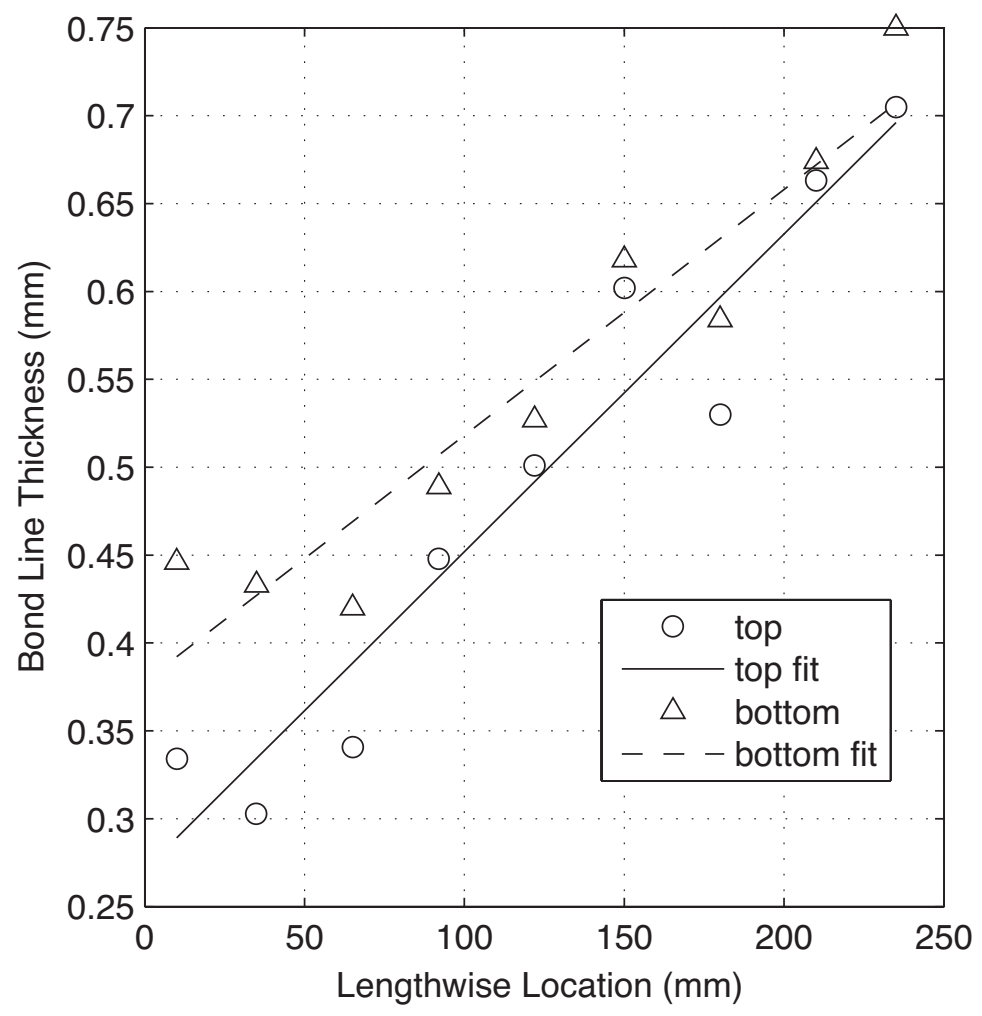

Figure 4: Calibration specimen thickness sample measurements. The origin of the plot is at the specimen edge near the PP tape. 


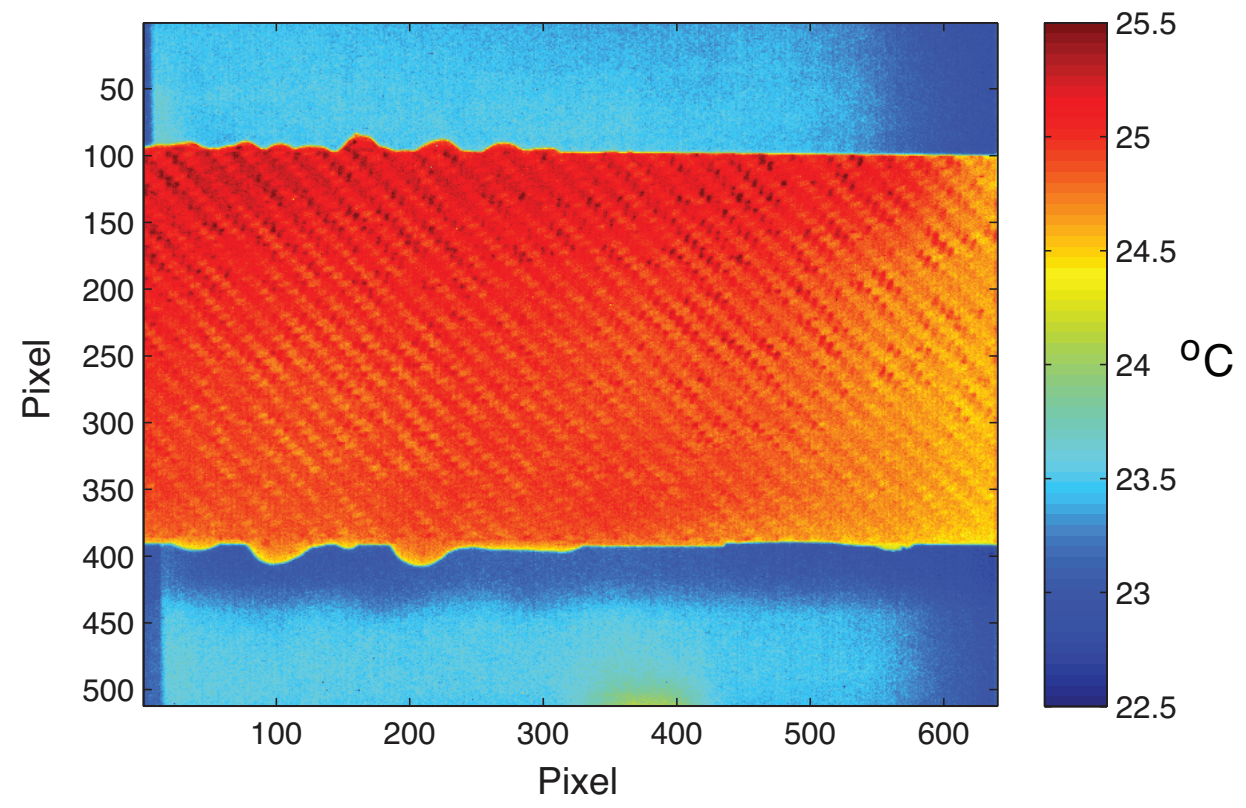

Figure 5: Thermal image of specimen and surrounding region $26 \mathrm{~s}$ after end of heat pulse. 




Figure 6: Phase contrast image of calibration specimen at maximum contrast. Defects from left to right are: PP, 1 layer PTFE, 4 layer PTFE, 2 layer PTFE. 


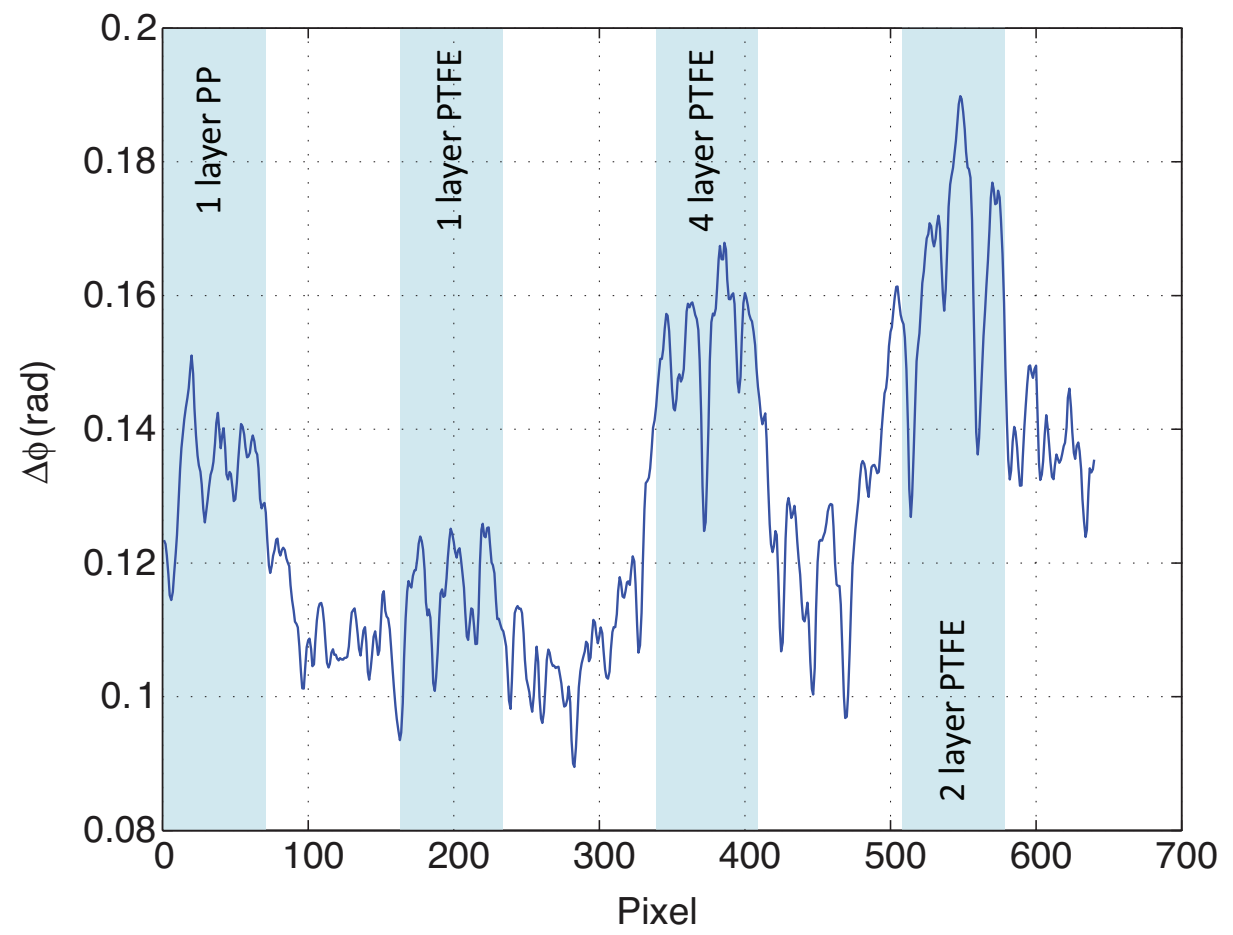

Figure 7: Phase contrast distribution across all defects (following red line in figure 6). 




Figure 8: Calculated damage locations for two center defects of calibration sample applying $72.6 \%$ of maximum defect phase contrast criterion. Actual boundaries of defects are plotted in red. 
(a)

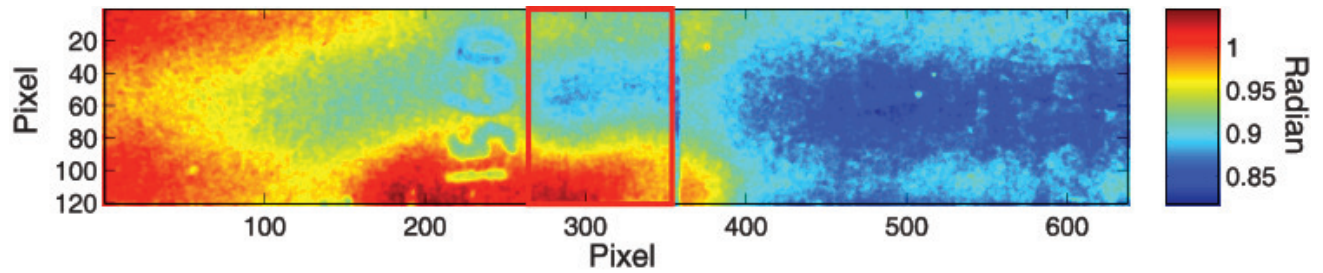

(b)


(c)
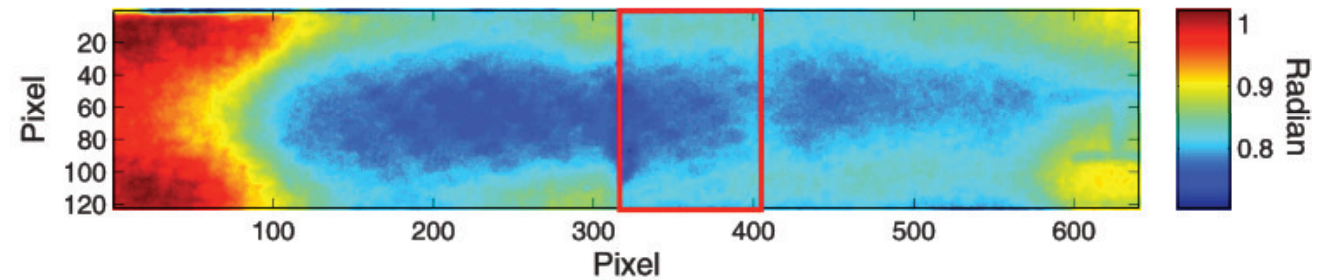

Figure 9: Calculated phase images from PPT of samples (a) 1, (b) 2 and (c) 3 after fabrication $\left(f_{s}=6 \mathrm{~Hz}, N=196, f=0.1 \mathrm{~Hz}\right)$. Boundaries of adhesive bond region is shown by red box. Figure 4.4 (a) - (c). 


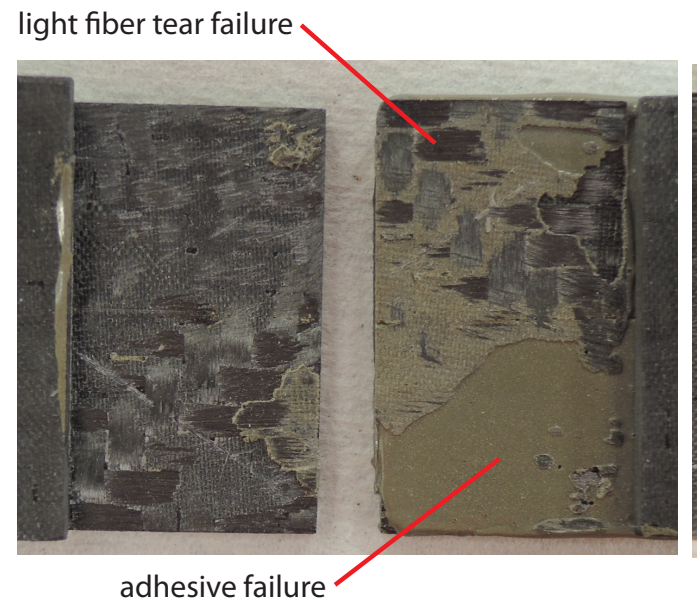

(a)

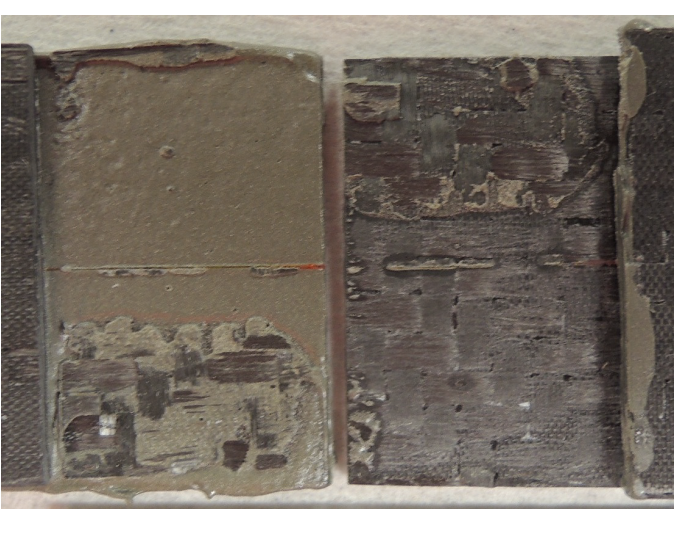

(b)

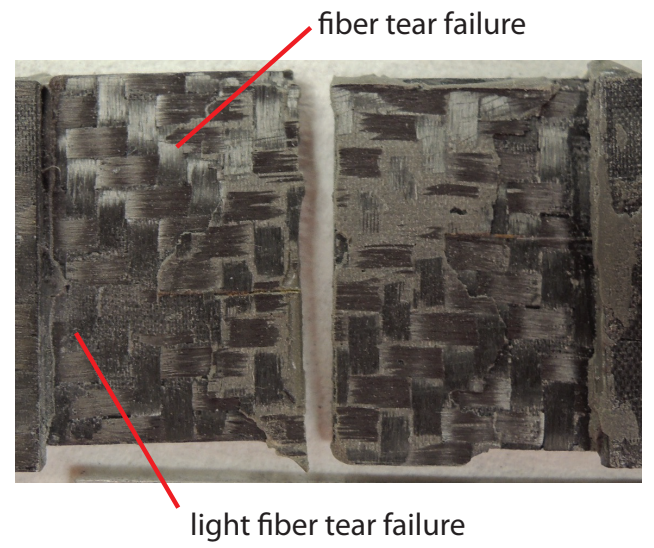

(c)

Figure 10: Failure surfaces of adhesively bonded joint for specimens (a) 1, (b) 2, and (c) 3. 


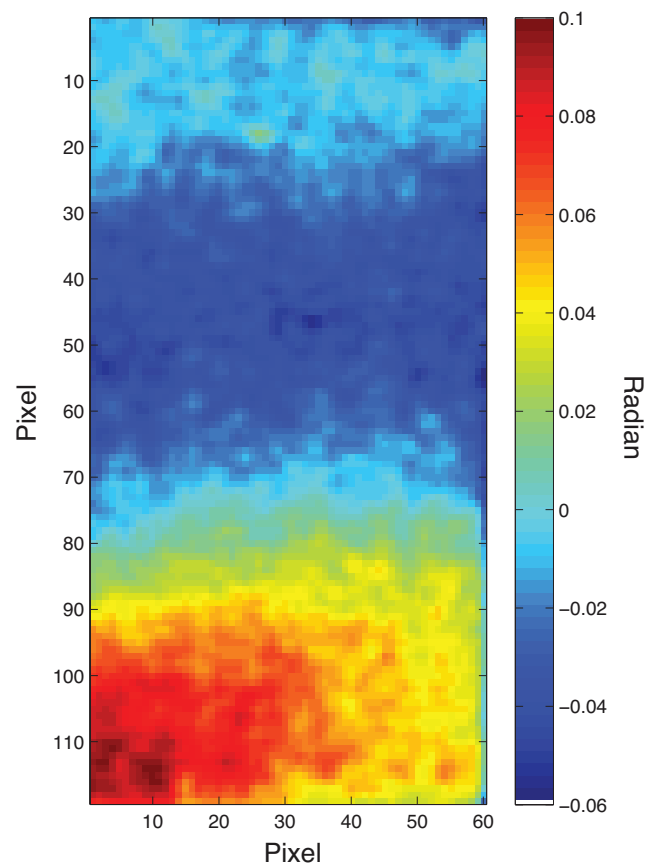

(a)



(b)

Figure 11: (a), (b) Phase contrast images of specimen 1 adhesive bond region, taken immediately after fabrication, using two separate user defined regions of minimum phase values. 

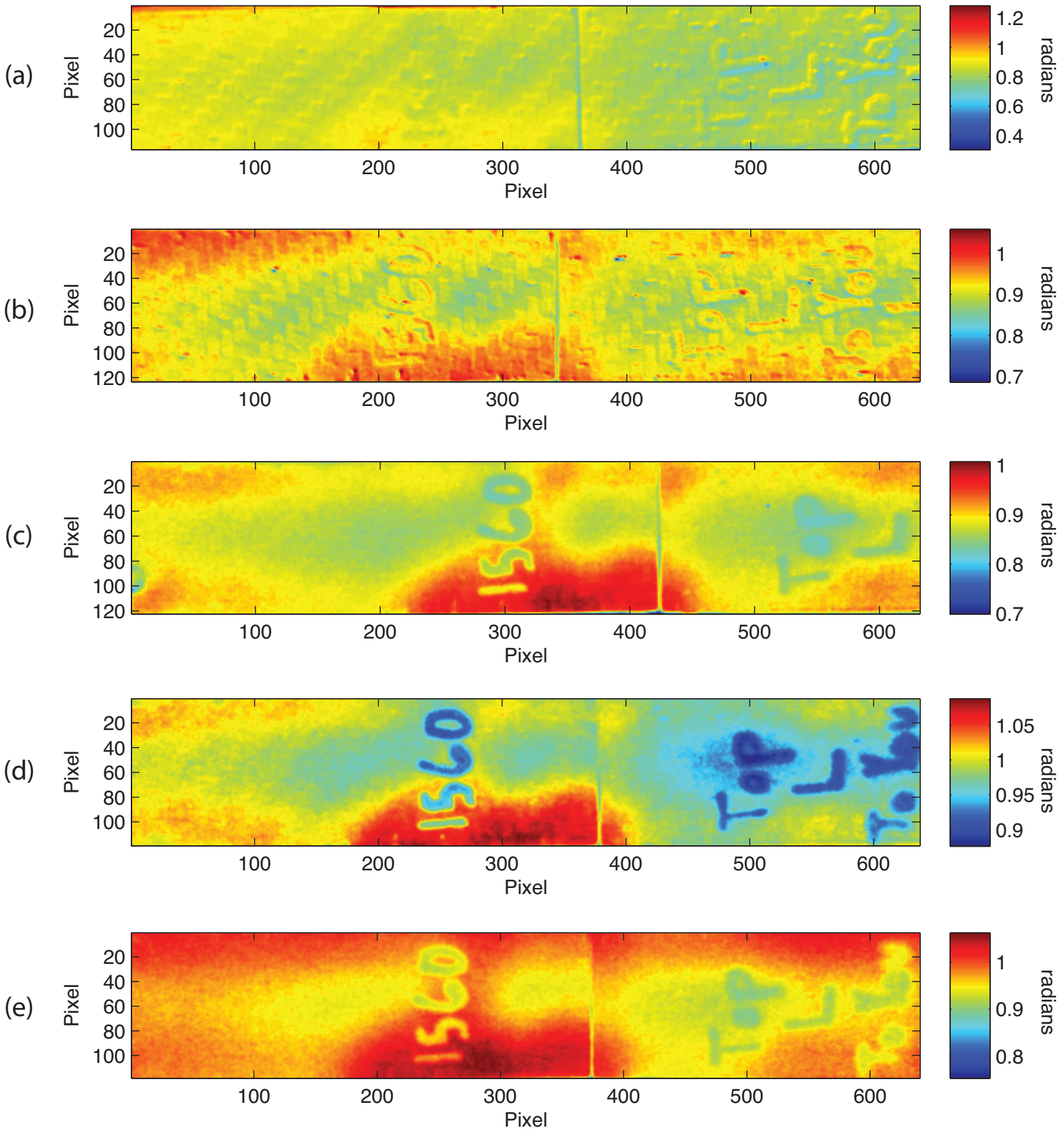

Figure 12: Phase image of Sample $1\left(f_{s}=6 \mathrm{~Hz}, N=196, f=0.1 \mathrm{~Hz}\right)$ after: (a) 200 cycles; (b) 600 cycles; (c) 600 cycles and pre-tension; (d) 1000 cycles; (e) 1600 cycles. 

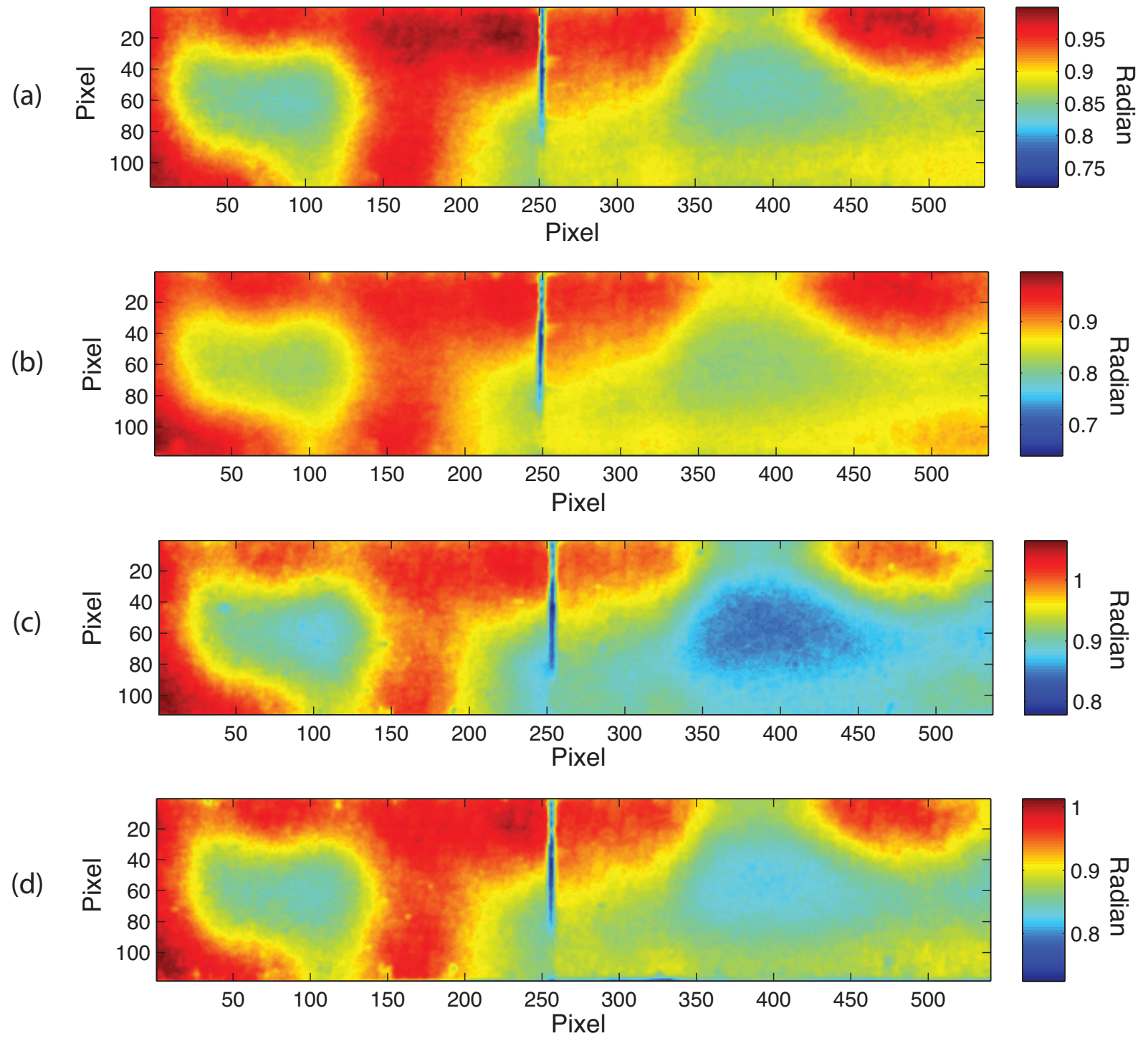

Figure 13: Phase image of Sample $2\left(f_{s}=6 \mathrm{~Hz}, N=196, f=0.1 \mathrm{~Hz}\right)$ after: (a) 200 cycles; (b) 400 cycles; (c) 400 cycles and pre-tension; (d) 600 cycles. 
(a)
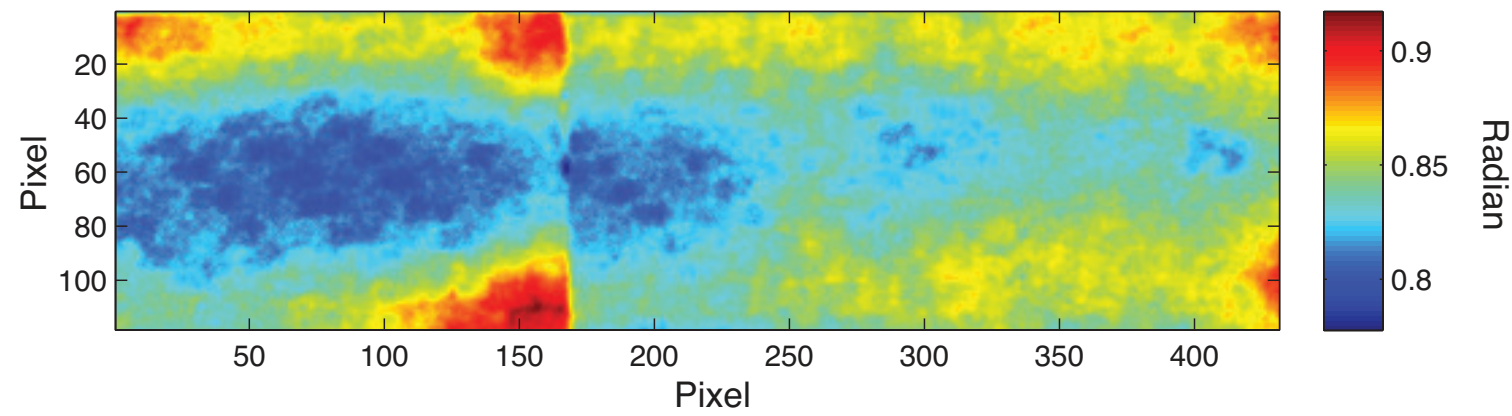

(b)
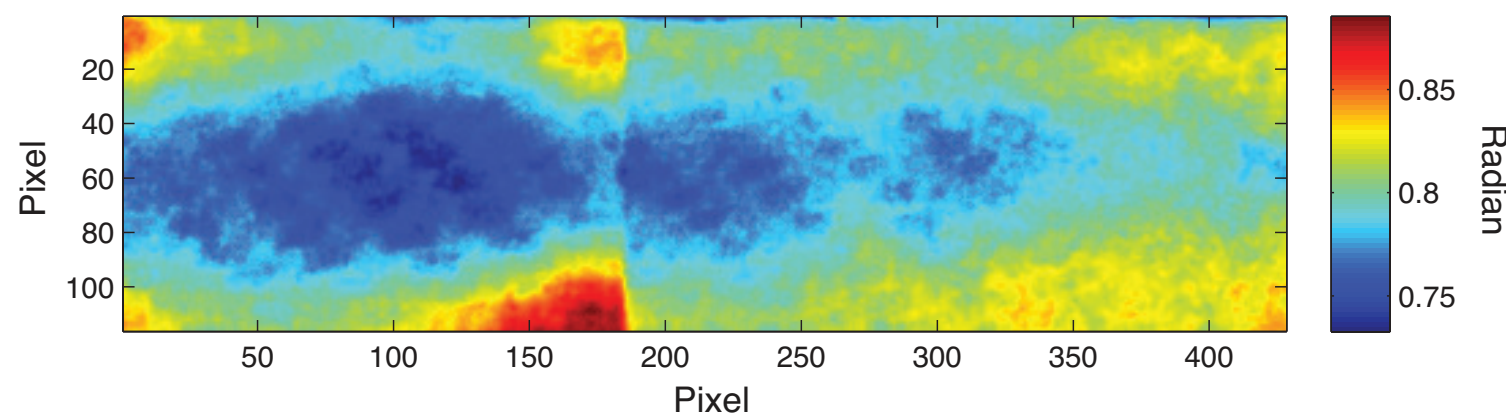

(c)
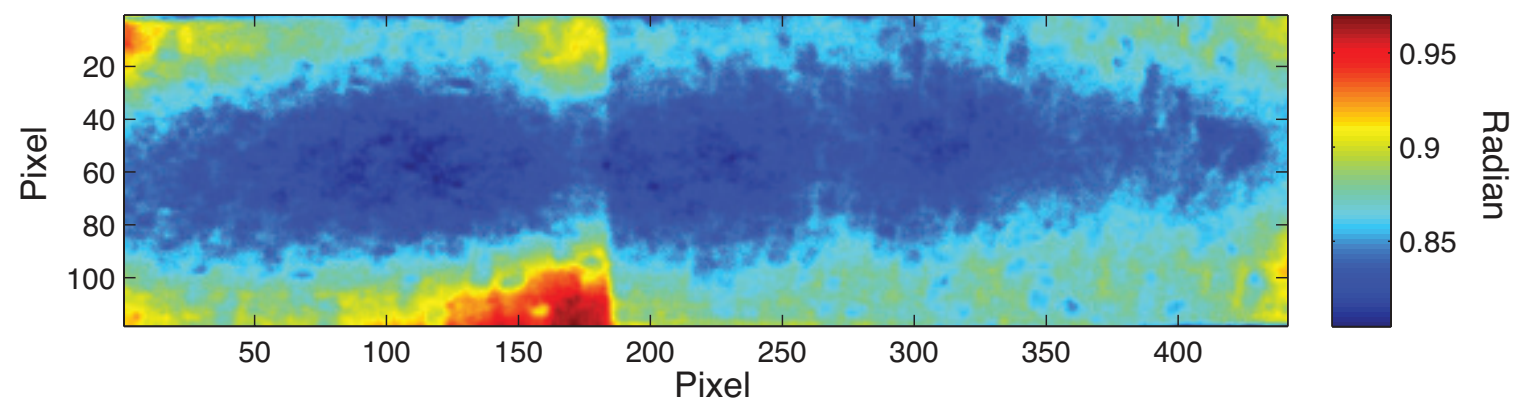

Figure 14: Phase image of Sample $3\left(f_{s}=6 \mathrm{~Hz}, N=196, f=0.1 \mathrm{~Hz}\right)$ after: (a) 1000 cycles; (b) 4000 cycles; (c) 50000 cycles. 


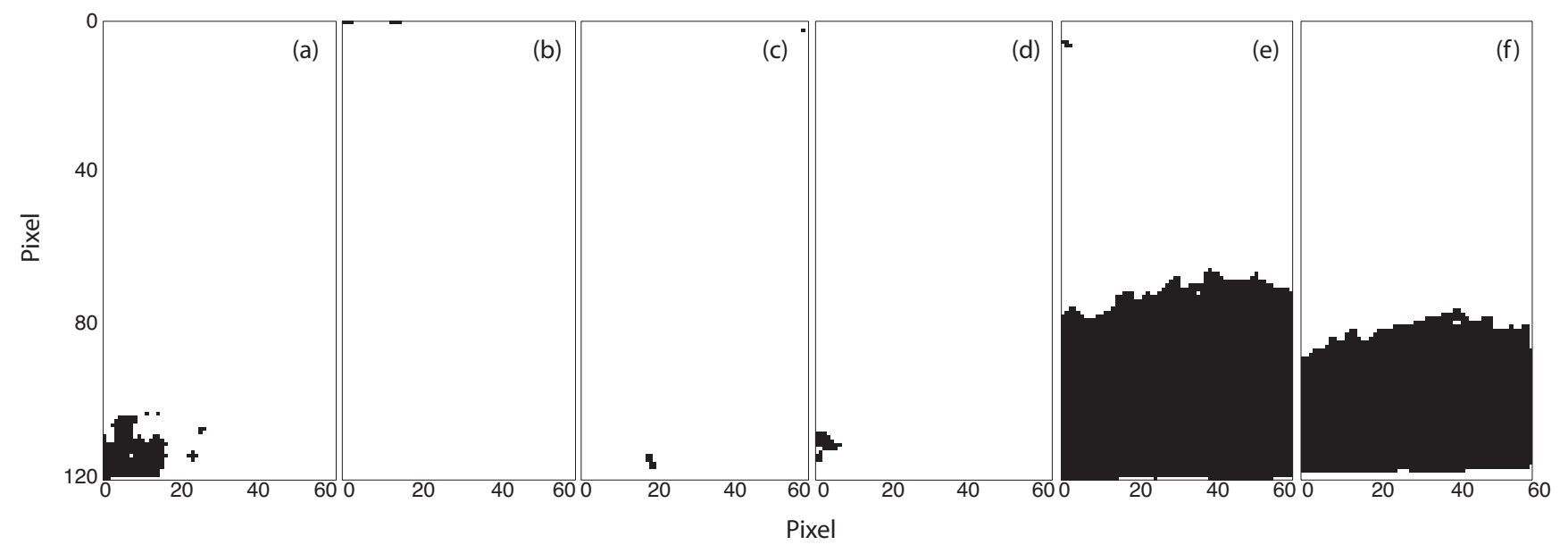

Figure 15: Binary image expression of phase contrast value in Sample 1 after applying threshold value 0.66 of maximum phase value after: (a) 0 cycles; (b) 200 cycles; (c) 600 cycles; (d) 600 cycles and pre-tension; (e) 1000 cycles; (f) 1600 cycles. 


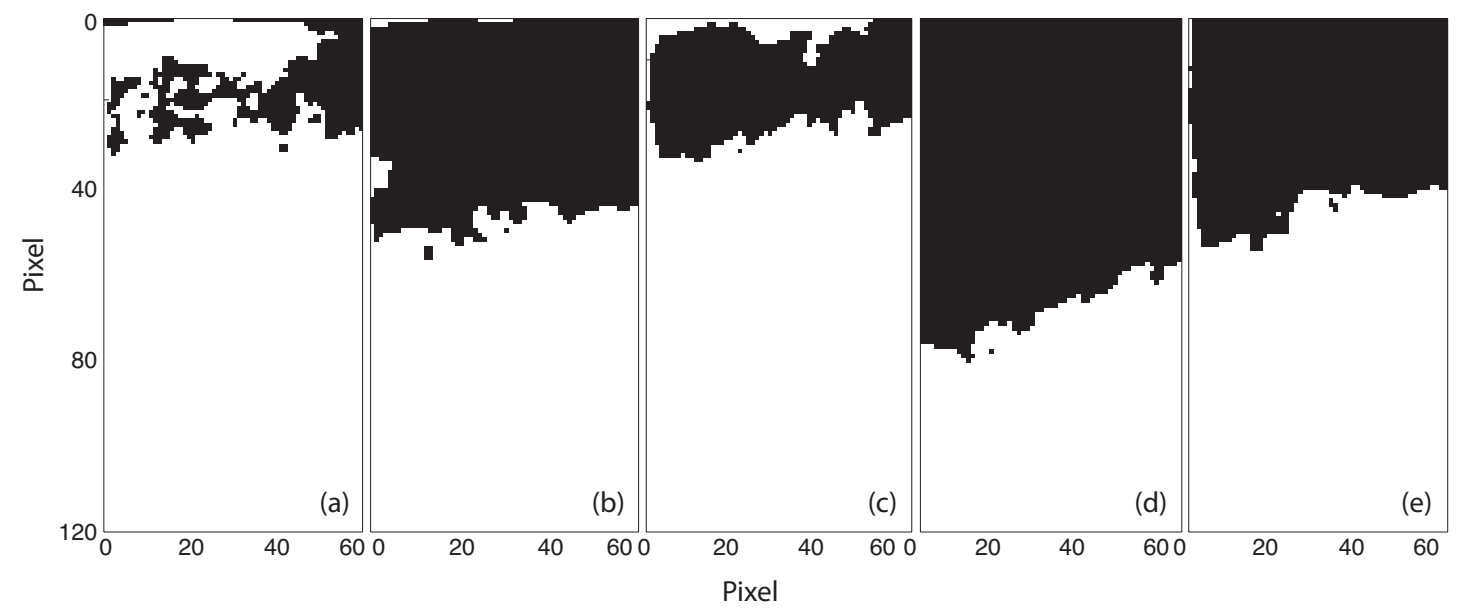

Figure 16: Binary image expression of phase contrast value in Sample 2 after applying threshold value 0.66 of maximum phase value after: (a) 0 cycles; (b) 200 cycles; (c) 400 cycles; (d) 400 cycles and pre-tension; (e) 600 cycles. 


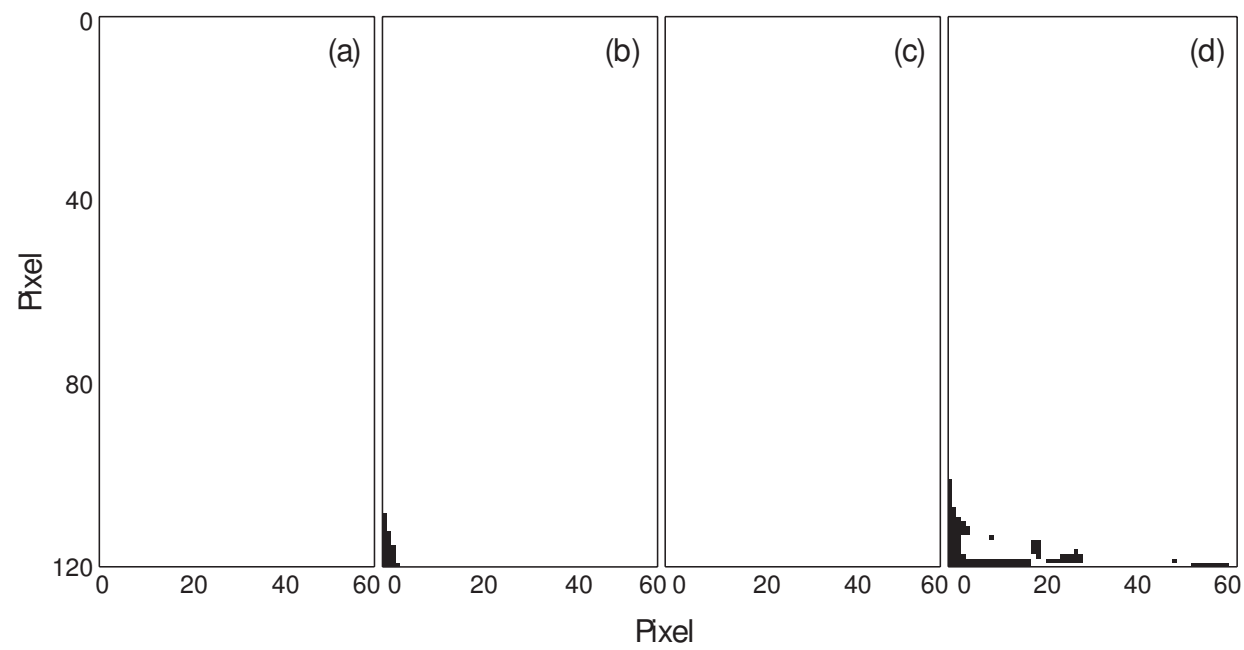

Figure 17: Binary image expression of phase contrast value in Sample 3 after applying threshold value 0.66 of maximum phase value after: (a) 0 cycles; (b) 1000 cycles; (c) 4000 cycles; (d) 500000 cycles. 